\title{
Research on Parking Location Problems for Bicycle-sharing under Shared Economic Background
}

\author{
Sheng Zhong ${ }^{a}$, Susu Chang ${ }^{b}$ and Shiming Yuan ${ }^{c}$ \\ School of business, Sichuan University, Chengdu 610000, China. \\ aszhong666@163.com, bcssscu@126.com, cysmingscu@163.com
} Keywords: Bicycle-sharing, Location Problems, Markov Process and LaGrange and Heuristic
Algorithm.

\begin{abstract}
The bicycle-sharing is the latest business model currently. How to reasonably select the parking site is the key factor for diversified products occupying the market. Random change process concerning the quantity of bicycles in the parking site is the unique attribute. Different from the traditional location model, the article uses the Markov process for modeling of dual-index, namely serving and coverage capabilities of the parking site, the LaGrange and heuristic algorithm to solve the parking site of bicycles and also the Matlab program for analog computation and analyses the experimental results.
\end{abstract}

\section{Introduction}

As a new pattern of sharing economy, the bicycle-sharing refers to a business model for daily "last $3 \mathrm{~km}$ " traffic pain point of residents. From 2007 to 2014, the bicycle-sharing has been mainly based on the bicycles with piles. The fixed location for taking and returning bicycles has increased the complication degree of bicycle utilization without solving the problem of "last $1 \mathrm{~km}$ " resulting in low usage rate and flow rate of bicycles with piles. Along with the fast development of mobile Internet, in 2014, the Internet-based bicycle-sharing led by OFO emerged at the right moment and more convenient bicycles without piles started to replace the bicycles with piles. Since the end of 2016, the "Internet plus" bicycle-sharing represented by "OFO bicycle" and "Mobike" become hot in major cities solving the "last $1 \mathrm{~km}$ " problem regarding the urban public transportation system.

The "Internet plus" bicycle-sharing brings not only great convenience for people but also new problems that the supervion on bicycle-sharing according to policies and regulations is still in the blank state with incomplete market management, serious disorderly parking of bicycles and more difficult management on urban space. With the fast development of bicycle-sharing, corresponding administrative rules are required to gradually standardize the urban parking site of bicycles. The article regards the parking site of bicycles as a service station and uses the coverage model and Markov process to study the coverage capability at the parking site and circulation and stability of bicycles there different from the location research on traditional service station. The parking site shall be selected scientifically to improve the use ratio of bicycles and their service ability at the site, reduce the later artificial management expense and optimize the economic sharing benefit.

The research on the location problems originated very early, and the literatures[1-7]are the domestic and overseas review literatures related to location introducing the overall development and conclusion of location; and as one of typical location models, the MCLP (namely the maximum covering location problem) has wide application prospect in the location. In the practical application, MCLP model is mainly used to solve the location problems of emergency service facilities. The MCLP model is used in the literature [8] studying the location problems of medical resources in remote and border areas; and it is applied in the literatures $[9,10]$ for the location problems of realistic emergency medical vehicles. The application of coverage model in the site selection and planning of emergency service facilities is reviewed in the literature [11]. Based on the Markov process, the warehouse location problems are studied in the literature [12].

In the existing literature researches, there are few researches on site selection for public facilities, terminal corporate service stations, etc. In the site selection for terminal services sites of bicycle- 
sharing enterprises, the serving capability, response speed and coverage scope are important factors to obtain the market share. Different from traditional service station, the parking site of bicycles covers small area, which cannot be used as the absolute decision factor; According to above contents, the article proposes a mathematic site selection model based on dual-index of covering and serving abilities for the economic sharing features, and uses the LaGrange and heuristic algorithm for calculation methods.

\section{Establishment of Models}

\subsection{Serving capability evaluation function}

Considering the site selection features of bicycle-sharing, in order to avoid the one-way flow of bicycles in the parking site resulting in the vehicle gathering or loss there, the article uses the Markov process to study the parking site. The changes of vehicle quantity at the parking site (in the course of parking and use) are regarded as a random fluctuation, so as to constitute the Markov process with discrete time and state. It is assumed that the quantity of all bicycles in the initial state at the parking site is $\delta$, and the state is $\tau_{n}, n=0,1,2,3 \ldots$ after recording the nth fluctuation if not considering the time factors. During the data collection stage, it is assumed that the total quantity of bicycles at each parking site exceeds 0 and $\delta$ in the spatial state of sample is regarded as the null point, namely the initial state of $\tau_{0}=0$, to obtain the sample space of $\rho_{1}(-n, 1-n \ldots,-2,-1,0,1,2, \ldots, n-1, n)$ and one bicycle added in the service station as per the probability $p$ and one bicyle lessed as per the probability $q$, where $p+q=1$ and $\mu_{n}=\sum_{k=1}^{n} \gamma_{k}$, representing the average bicycle quantity at the parking site after $N$ steps with $\gamma_{k}$ referring to each change of bicycle.

$P\left\{\gamma_{k}=1\right\}=p, P\left\{\gamma_{k}=-1\right\}=q, k=1,2, \ldots, n$

$E\left\{\gamma_{k}\right\}=P\left\{\gamma_{k}=1\right\} * 1+P\left\{\gamma_{k}=-1\right\} *(-1)=p-q$

$E\left\{\mu_{n}\right\}=E\left\{\sum_{k=1}^{n} \gamma_{k}\right\}=\sum_{k=1}^{n} E\left\{\gamma_{k}\right\}=n(p-q)$

$E\left\{\mu_{n}\right\}$ shows the average bicycle quantity at the parking site after $N$ steps; and the mass point reaching $\tau_{n}=m$ after $N$ steps indicates that the mass point fluctuates $\frac{n+m}{2}$ steps forwards and $\frac{n-m}{2}$ steps backwards, so as to obtain the one-dimensional state quantity distribution;

$$
P\left(\tau_{n}=m\right)=\left(\begin{array}{c}
n \\
\frac{m+n}{2}
\end{array}\right) p^{\frac{n+m}{2}} q^{\frac{n-m}{2}}: \begin{gathered}
m=n,-n+2,-n+4, \ldots \ldots, n-2, n ; m \\
\leq n
\end{gathered}
$$

When $m \in\left[-\frac{\delta_{0}}{2}, \frac{\delta_{0}}{2}\right]\left(\delta_{0}=\left|E\left\{\mu_{n}\right\}\right|\right.$ refers to the quantity of bicycles at the parking site in the initial state), the parking site is regarded in the stable state with the probability $P_{j n}$ of mass point in the stable state as the serving capability evaluation function of such parking site;

$$
\begin{aligned}
& P_{j n}=\sum_{i=-\frac{\delta_{0}}{2}}^{\frac{\delta_{0}}{2}} P\left(\tau_{n}=j\right) \\
& =\sum_{j=-\frac{\delta_{0}}{2}}^{\frac{\delta_{0}}{2}}\left(\frac{n}{2}\right) p^{\frac{n+j}{2}} q^{\frac{n-j}{2}} ; \quad j=n,-n+2,-n+4, \ldots \ldots, n-2, n ; j \leq n
\end{aligned}
$$

\subsection{Coverage capability function}

The bicycle-sharing coverage capability at the parking site is determined by the distance of demand point. Based on the response speed of service station measured by the time satisfaction function in the literature [13], it is assumed that $t_{i j}$ is the shortest route for potential site selection $j$ of demand point $i, L_{i}$ is the longest route for satisfied evaluation on potential site selection of demand point $i$, and $U_{i}$ is the shortest route for very unsatisfied evaluation on potential site selection of demand point $i$. Where $L_{i} \leq U_{i}, f\left(t_{i j}\right)$ refers to the satisfaction degree of demand point $i$ to the coverage capability of service station $j$. 


$$
f\left(t_{i j}\right) \begin{cases}1, & t_{i j}<L_{i} \\ 1-\left(\frac{t_{i j}-L_{i}}{U_{i}-L_{i}}\right)^{k} & t_{i j} \in\left[L_{i}, U_{i}\right] \\ 0, & t_{i j}>U_{i}\end{cases}
$$

Where, $k>0$ is the sensitivity coefficient When $t_{i j} \in\left[L_{i}, U_{i}\right]$ and $k<1, f\left(t_{i j}\right)$ is the concave function; when $k>1, f\left(t_{i j}\right)$ is the convex function; and when $k=1, f\left(t_{i j}\right)$ is the straight line. Obviously $f\left(t_{i j}\right) \in[0,1]$, we regard the function as the coverage capability evaluation function of potential site selection to the demand point.

In the given network $G(V, A), V$ is the fixed-point set with $|V|=n$ and $A$ as the frontier set. For $i \in I$ and $j \epsilon J$, I and $J$ are respectively the index sets of latent demand and alternative site set with $I \cup$ $J=V . s_{i}$ is the demand quantity of demand point $i$ and $P$ is the quantity of service state which shall be planned in such network. $f\left(t_{i j}\right)$ Refers to the satisfaction evaluation on coverage level of the customer at the demand point $i$ to the potential site selection $j$. We select the whole under the dualindex of coverage and serving capabilities as the optimal solution.

It is shown as the model $P 1$ :

$$
\text { s.t: } \begin{cases}\sum_{j \in J} Y_{i j}=1 & \forall i \in I \\ \sum_{j \in J} X_{j}=p & \\ Y_{i j}-X_{j} \leq 0 & \forall i \in I, j \in J \\ X_{j}=0,1 & \forall i \in I \\ Y_{i j}=0,1 & \forall i \in I, j \in J\end{cases}
$$

When $X_{j}=1$, the potential point $j$ is selected as the site point, otherwise $X_{j}=0$; and if the demand point $i$ is covered at the potential point $j, Y_{i j}=1$, otherwise $Y_{i j}=0$. For the problem of $P 1$, the objective functional expression (5) shows the optimal solution under the double weights; the constrained expression (6) indicates that each demand point $i$ only selects the service at the closest site point; the constrained expression (7) indicates that the quantity of site points set preliminarily is $p$; the constrained expression (8) indicates whether the set of site point will restrain the objective function and if the potential point is not selected, the demand point covered in the potential point will not influence the objective function; and the constrained expressions (9) and (10) show that the decision variable is restrained as $(0,1)$.

\section{Analyses for LaGrange an Heuristic Algorithm}

There are lots of algorithms to solve the location problems of facilities, and the LaGrange and heuristic algorithm is one of them with high efficiency in solving the discrete type site selection. The basic principle of the LaGrange and heuristic algorithm is gradually removing or broadening the constraint conditions causing difficulties in solving the objective function and then absorbing these constraint conditions into the objective function by penalty parameters or Lagrange's multiplier. After the changes, the objective function still keeps linear, and former problem will be solved more easily after the difficulty constraints are absorbed. The literatures [14-16] introduce the application of the LaGrange and heuristic algorithm in site selection and optimization of combination.

According to the relaxation principle, we relax the constrained expression (6) in the problem $P 1$ into the objective function (5) to obtain:

$$
\begin{aligned}
& \operatorname{Max} \sum_{i \in I} \sum_{j \in J} s_{i}\left[\omega_{i j} f\left(t_{i j}\right)+\omega_{j} P_{j n}\right] Y_{i j}+\sum_{i \in I} \lambda_{i}\left(1-\sum_{j \in J} Y_{i j}\right) \\
& =\operatorname{Max} \sum_{i \in I} \sum_{j \in J}\left(s_{i}\left[\omega_{i j} f\left(t_{i j}\right)+\omega_{j} P_{j n}\right]-\lambda_{i}\right) Y_{i j}+\sum_{i \in I} \lambda_{i}
\end{aligned}
$$

s.t: $\quad$ constrains(7), (8), (9)and(10) 
Obviously, for the problem $P 2$, we can obtain its optimal solution and optimal value. When $\lambda_{i}$ is non-negative, the optimal solution of $P 2$ is the upper limit of the optimal value of problem $P 1$, taking as $Z_{U}$. In addition, $J^{*}$ is defined as the index set of the optimal solution of problem $P 2\left(X_{j}=1\right)$, taking $J^{*}=\left\{j / X_{j}=1\right\}$.

$Z_{L}$ is defined as the lower limit of the optimal solution of problem $P 2$; and in the problem $P 2$, the constrained expression (6) is relaxed to the objective function and the result cannot be determined as the optimal solution of problem $P 1$. However, for any demand point $i$, the potential point with the optimal dual evaluation $\varphi_{i}$ on time satisfaction and stable potential point under the problem $P 2$, namely:

$$
Z_{L}=\sum_{j \in J^{*}} \varphi_{i}=\sum_{j \in J^{*}} \max \left\{\left(s_{i}\left[\omega_{i j} f\left(t_{i j}\right)+\omega_{j} P_{j n}\right]\right\}\right.
$$

According to above conclusions, we can obtain the upper and lower limits of the optimal value of problem $P 1$, namely $Z_{U}$ and $Z_{L}$ respectively. Then the sub gradient algorithm is used to approach the optimal solution. $k$ represents the iterations, $Z_{U}^{k}$ and $Z_{L}^{k}$ respectively indicate the upper and lower limits of the optimal value after the kth iterations, and $\lambda_{i}^{k}$ represents the corresponding Lagrange multiplier; $X_{j}^{k}$ and $Y_{i j}^{k}$ are the optimal solution of problem P2; $t^{k}$ indicates the iteration step length, $L B$ shows the the maximum lower limiting value after the stop of kth iterations, $U B$ shows the the minimum lower limiting value after the stop of kth iterations, and $\alpha^{k}$ refers to the parameters of the kth iteration step length. Where,

$$
\begin{aligned}
& t^{k}=\frac{\alpha^{k}\left(Z_{U}^{k}-L B\right)}{\sum_{i \in I}\left(1-\sum_{j \in J} Y_{i j}^{k}\right)^{2}} \\
& \quad \lambda_{i}^{k+1}=\max \left\{0, \lambda_{i}^{k}-t^{k}\left(1-\sum_{j \in J} Y_{i j}^{k}\right)\right\} \quad \forall i \in I
\end{aligned}
$$

If any one of the following four conditions is set, the iteration is terminated:

(1) $\sum_{j \in J} Y_{i j}=1 \quad \forall i \in I ;(2) U B-L B \leq 0.3 ;(3) t^{k} \leq 0.0001 ;(4) k=400$.

\section{Computational Experiments}

For the convenience of computation, we use the Mat lab program to calculate the model; and the original value is set for each parameter of the model as follows. We consider the number of demand points as 200, 400, 600, 800 and 1,000; the demand quantity of demand points is generated randomly as per the density of normal distribution $N\left(200,100^{2}\right)$, and the distance between the demand points and potential points is generated randomly as per the density of normal distribution $N\left(130,70^{2}\right)$; $L_{i} \forall_{i} \in I$ is generated evenly and randomly within $[10,100], U_{i}=L_{i}+d_{i} \forall_{i} \in I, d_{i} \forall_{i} \in I$ is generated evenly and randomly within $[10,50]$, and the sensitivity coefficient $k_{i} \forall_{i} \in I$ is generated evenly and randomly within $[0.5,1.5]$; the above random numbers remain non-negative; the sample space $\rho_{1}$ conforms to the Poisson distribution with the same parking and taking probabilities; in the expression (3), $n=50$; in the expression (11), $\omega_{i j}=\omega_{j}=0.5 \forall_{i} \in I, \forall_{i} \in J$; in the expresions (13) and (14), the initialization parameter $k=1 ; U B \leftarrow+\infty ; L B \leftarrow-\infty$; and $\lambda_{i}^{1} \leftarrow \bar{s}+1 / 2$ (s $\left.\mathrm{s}_{\mathrm{i}}\right) \quad \forall_{i} \in I, \alpha^{k} \leftarrow 2$.

Figure 1 shows the difference between site selection results under single and double indexes when $(n, m, p)=(400,30,15)$, representing the influence on the decision for parking site under the consideration of serving capability index. Due to the length of the article, operating results are not post. The operating results under different input parameters indicates the increase of objective function value along with the increase of quantity in demand points, potential points and site points satisfying the realistic rule due to different increments. From Figure 2, solving this model by LaGrange an heuristic algorithm has better efficiency with the mean deviation of upper and lower limiting value at about $2.3 \%$. Due to the great difficulties in obtaining real data and many parameters involved in the model and randomly generated, there is certain error in solving the real data. However, based on a large amount of data, after adjusting the search parameters of the algorithm, an optimal solution can be obtained. 


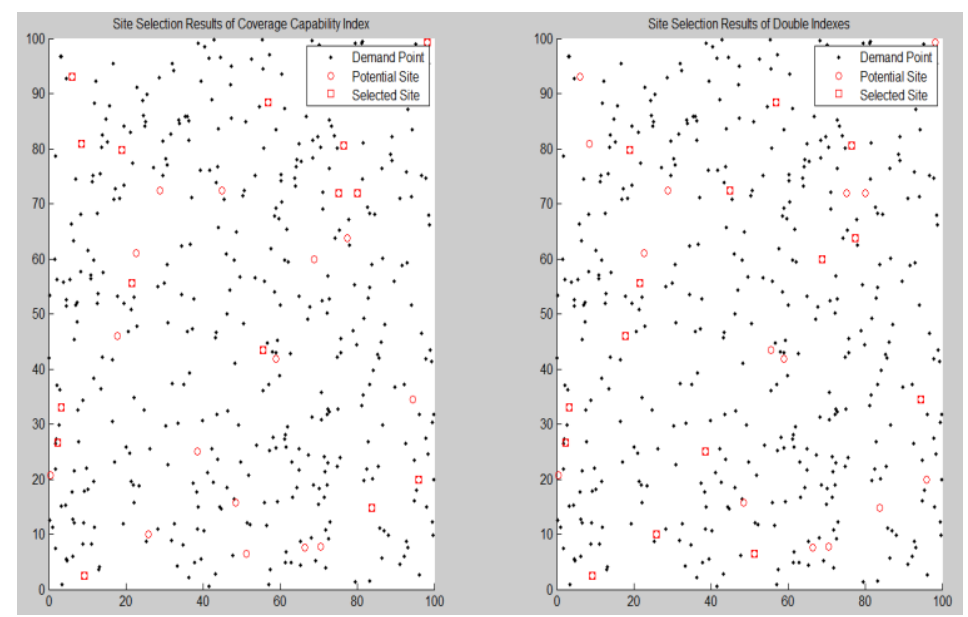

Figure 1. Comparison Diagram for Site Selection Results of Single and Double Indexes

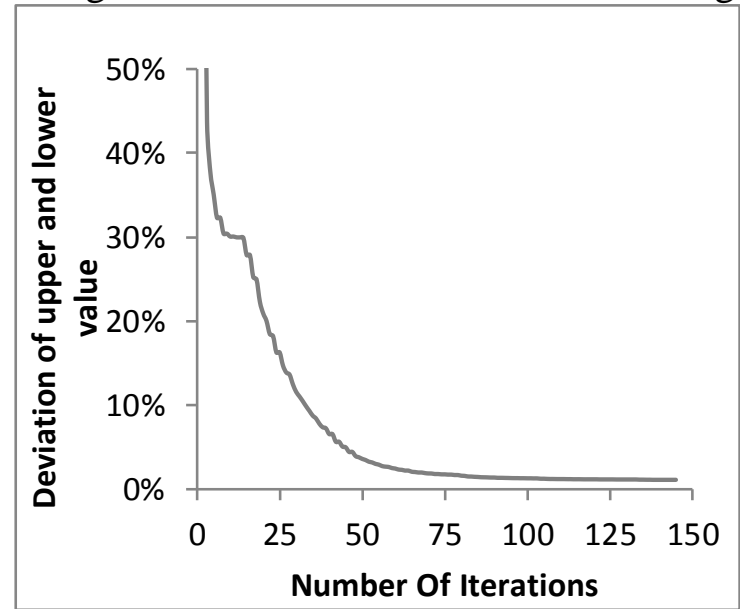

Figure 2. Model Convergence when $m=400, n=50$ and $p=15$

\section{Summary}

The article establishes the serving capability evaluation function and coverage capability function model, selects the best bicycle-sharing parking site through the LaGrange an heuristic algorithm, provides the theoretical basis for the determination of bicycle-sharing parking site, and determines the large-scale parking layout from the whole, which is in favor of the reasonable use of resources. Of course, multiple problems exist for further discussion and research, such as the data involved in the article generated randomly by computer basically with insufficient authenticity; the Markov model used for further research on the flow rate of bicycles between regions; more analysis for the serving capability of parking site according to the population density and traffic conditions at the parking site and determination of bicycle quantity there, which will be our future research emphasis.

\section{References}

[1]. Tansel, B.C., R.L. Francis and T.J. Lowe, Location on networks - a survey .1. The p-center and p-median problems. Management Science, 1983. 29(4): p. 482-497.

[2]. Tansel, B.C., R.L. Francis and T.J. Lowe, Location on networks - a survey .2. Exploiting tree network structure. Management Science, 1983. 29(4): p. 498-511.

[3]. Hale, T.S. and C.R. Moberg, Location science research: a review. Annals of Operations Research, 2003. 123(1-4): p. 21-35.

[4]. Revelle, C.S. and H.A. Eiselt, Location analysis: a synthesis and survey - invited review. European Journal Of Operational Research, 2005. 165(1): P. 1-19. 
[5]. Wang Fei, Xu Yu and Li Yixue, Review on facility location models, Operations Research And Management Science, 2006(05): p64-69.

[6]. Yang Fengmei et al., Some advances of the researches on location problems, Operations Research and Management Science, 2005(06): p1-7.

[7]. Qiao Lianbao, Classification and review on the covering facility location problem, Logistics SciTech, 2015(03): p59-66

[8]. Bennett, V.L., D.J. Eaton and R.L. church, selecting sites for rural health-workers. Social Science \& Medicine, 1982. 16(1): p. 63-72.

[9]. Eaton, D.J., et al., determining emergency medical-service vehicle deployment in Austin, texas. interfaces, 1985. 15(1): p. 96-108.

[10]. Eaton, D.J., et al., Determining ambulance deployment in santo-domingo, dominican-republic. Journal Of The Operational Research Society, 1986. 37(2): p. 113-126.

[11]. Li, X., et al., Covering models and optimization techniques for emergency response facility location and planning: a review. Mathematical Methods Of Operations Research, 2011. 74(3): p. 281-310.

[12]. Mirchandani, P.B., Locational decisions on stochastic networks. Geographical Analysis, 1980. 12(2): p. 172-183.

[13]. Ma Yunfeng et al., Maximum covering location problems based on time satisfaction, Chinese Journal Of Management Science, 2006(02): p45-51

[14]. Sridharan, R., A heuristic lagrangean algorithm for the capacitated plant location problem - a comment. European Journal Of Operational Research, 1986. 23(2): p. 264-265.

[15]. Agar, M.C. and S. Salhi, Lagrangean heuristics applied to a variety of large capacitated plant location problems. Journal Of The Operational Research Society, 1998. 49(10): p. 1072-1084.

[16]. Karasakal, O. and E.K. Karasakal, A maximal covering location model in the presence of partial coverage. Computers \& Operations Research, 2004. 31(9): p. 1515-1526. 